\title{
Analysis and Design of Networked Control Systems with Random Markovian Delays and Uncertain Transition Probabilities
}

\author{
Li Qiu, ${ }^{1}$ Chengxiang Liu, ${ }^{2}$ Fengqi Yao, ${ }^{3}$ and Gang Xu ${ }^{1}$ \\ ${ }^{1}$ Shenzhen Key Laboratory of Urban Rail Transit, College of Mechatronics and Control Engineering, Shenzhen University, \\ Shenzhen 518060, China \\ ${ }^{2}$ Shenzhen Key Laboratory of Laser Engineering, College of Electronic Science and Technology, Shenzhen University, \\ Shenzhen 518060, China \\ ${ }^{3}$ School of Electrical Engineering and Information, Anhui University of Technology, Maanshan 243000, China
}

Correspondence should be addressed to Gang Xu; xugang@szu.edu.cn

Received 16 January 2014; Accepted 3 March 2014; Published 14 April 2014

Academic Editor: Shuping He

Copyright ( 2014 Li Qiu et al. This is an open access article distributed under the Creative Commons Attribution License, which permits unrestricted use, distribution, and reproduction in any medium, provided the original work is properly cited.

\begin{abstract}
This paper focuses on the stability issue of discrete-time networked control systems with random Markovian delays and uncertain transition probabilities, wherein the random time delays exist in the sensor-to-controller and controller-to-actuator. The resulting closed-loop system is modeled as a discrete-time Markovian delays system governed by two Markov chains. Using Lyapunov stability theory, a result is established on the Markovian structure and ensured that the closed-loop system is stochastically stable. A simulation example illustrates the validity and feasibility of the results.
\end{abstract}

\section{Introduction}

Networked control systems (NCS) find many successful applications in power grids, manufacturing plants, vehicles, aircrafts, spacecrafts, remote surgery, and so on [1]. Compared with the traditional control systems, the use of the communication networks brings many advantages such as low cost, reduced weight, and simple installation and maintenance, as well as high efficiency, flexibility, and reliability. However, inserting communication networks into feedback control loops has also resulted in several interesting and challenging issues, such as packet dropouts [2], time delays [3-10], quantization [11], time-varying transmission intervals [12], distributed synchronization [13], or some of the constraints considered simultaneously [14-17], which make the analysis and design of NCS complex. These imperfections block the way of harvesting reliable NCS by implementing existing control techniques [18]. To overcome these drawbacks, significant attention has been paid to the NCS research ranging from system identification and stability analysis to controller and filter designs. See the survey papers $[1,19,20]$ and the references therein.

The network-induced time delays are known to be the major challenges in NCS, which may be potential causes for the deteriorating performance or instability of NCS. Consequently, numerous works have been conducted on the time-delay issue in the past years. For example, in [21], the mixed $\mathrm{H}_{2} / \mathrm{H}_{\infty}$ control issue of NCS with random time delays has been investigated based on Markovian jump linear systems method. In [9], the stability problem of NCS with uncertain time-varying delays has been investigated. The stability and stabilization of NCS with random time delay usually use Markovian jump linear systems (MJLS) approach, and, recently, many significant achievements have been obtained for MJLS in [22-26]. However, most of the approaches for NCS based on Markovian jump systems framework assumed that the Markovian transition probabilities are known a priori, which severely limit the utility of the Markov model. Furthermore, such assumption may not hold true especially in the case where networked control is 
applied to the remote plants. Recently, the $H_{\infty}$ filter problem for a class of uncertain Markovian jumping systems with bounded transition probabilities has been investigated in [27], but the well-established results cannot be directly used to NCS. To the best of the authors' knowledge, up to now, very limited efforts have been devoted to studying the system with uncertain transition probability matrices for NCS, which motivates our investigation.

In this paper, we address the analysis and design of NCS with random time delays modeled by Markov chains in forward sensor-to-controller (S-C) and feedback controller-toactuator (C-A) communication links and with the uncertain transition probability matrices. The main contributions of this paper are highlighted as follows. (i) A model is proposed for NCS with random Markovian delays and uncertain transition probability matrices. (ii) The system modeled will be more generalized and avoid the ideal assumption that the transition probabilities are known a priori. (iii) New criteria for stability are obtained based on a Lyapunov approach. Finally, a numerical example is provided to demonstrate the effectiveness of the proposed control scheme for NCS with random time delays and uncertain transition probability matrices.

The remainder of this paper is organized as follows. A model with Markovian delays and uncertain transition probabilities is obtained in Section 2. The main results are obtained based on a Lyapunov approach and the linear matrix inequalities technique in Section 3. Section 4 presents the simulation results. Finally, the conclusions are provided in Section 5.

Notations. Matrices are assumed to have appropriate dimensions. $\mathbb{R}^{n}$ and $\mathbb{R}^{n \times m}$ denote the $n$-dimensional Euclidean space and the set of all $n \times m$ real matrices, respectively. The notations $A>0(A<0)$ indicate that $A$ is a real symmetric positive (negative) definite matrix. $I$ and 0 denote the identity matrix and the zero matrix with appropriate dimensions, respectively. Superscripts " $T$ " and " -1 " stand for the matrix transposition and the matrix inverse, respectively. $\mathbb{E}[\cdot]$ stands for the mathematical expectation and $\operatorname{diag}\{A, B\}$ stands for a block-diagonal matrix of $A$ and $B . I$ and 0 denote the identity matrix and zero matrix with appropriate dimensions, respectively. $\operatorname{sym}\{A\}$ denotes the expression $A+A^{T}$, and * means symmetric terms in symmetric entries.

\section{NCS Model}

The framework of networked control systems is depicted in Figure 1. The plant, sensor, controller, and actuator are spatially distributed and closed through a network. Random time delays exist in both of S-C and C-A.

The plant is described by the following discrete-time linear time-invariant plant model:

$$
x(k+1)=A x(k)+B u(k),
$$

where $x(k) \in \mathbb{R}^{n}$ is the system state vector and $u(k) \in \mathbb{R}^{m}$ is the control input. $A$ and $B$ are known real constant matrices with appropriate dimensions.

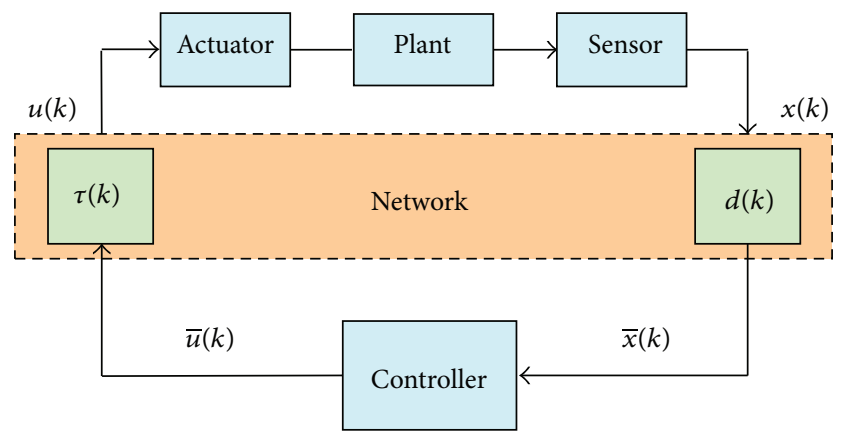

FIGURE 1: Diagram of a NCS with time delays.

For this system, we will consider a state feedback controller as follows:

$$
\bar{u}(k)=K \bar{x}(k),
$$

where $K$ is the state feedback controller gain.

Random S-C and C-A time delays are $d(k)$ and $\tau(k)$, respectively. $d(k)$ and $\tau(k)$ are assumed be bounded; that is, $0 \leq \underline{d} \leq d(k) \leq \bar{d}, 0 \leq \underline{\tau} \leq \tau(k) \leq \bar{\tau}$, where $\underline{d}=\min \{d(k)\}$, $\bar{d}=\max \{d(k)\}, \underline{\tau}=\min \{\tau(k)\}$, and $\bar{\tau}=\max \{\tau(k)\}$. One way to model delays $d(k)$ and $\tau(k)$ is by using the finite-state Markov chains presented in [21]. The main advantages of the Markov model considering the dependence between delays are that the current time delays in real networks delays are frequently related to the previous delays. In this paper $d(k)$ and $\tau(k)$ are modeled as two homogeneous Markov chains.

By substituting controller (2) to plant (1), we obtain a closed-loop system as follows:

$$
x(k+1)=A x(k)+B K x\left(k-\eta_{k}\right),
$$

where $\eta_{k}=\tau(k)+d(k-\tau(k))$.

In system (3), $\{d(k), k \in \mathbb{Z}\}$ and $\{\tau(k), k \in \mathbb{Z}\}$ are two finite state discrete-time homogeneous Markov chains with values in the finite sets $S_{1}=\left\{0, \ldots, s_{1}\right\}$ and $S_{2}=$ $\left\{0, \ldots, s_{2}\right\}$ with the uncertain transition probability matrices $\hat{\pi}$ and $\hat{\lambda}$. $\hat{\pi}=\left\{\hat{\pi}_{i j}\right\}$ and $\hat{\lambda}=\left\{\hat{\lambda}_{n m}\right\}$ denote the uncertain transition probability matrices of Markov chain $d(k)$ and $\tau(k)$, respectively, with probabilities $\widehat{\pi}_{i j}$ and $\hat{\lambda}_{n m}$, which are defined by

$$
\begin{gathered}
\operatorname{Pr}\{d(k+1)=j \mid d(k)=i\}=\widehat{\pi}_{i j}, \\
\operatorname{Pr}\{\tau(k+1)=n \mid \tau(k)=m\}=\widehat{\lambda}_{m n},
\end{gathered}
$$

where $\operatorname{Pr}\left\{\widehat{d}_{0}=i\right\}=\widehat{\pi}_{i} \geq 0, \operatorname{Pr}\left\{\widehat{\tau}_{0}=m\right\}=\hat{\lambda}_{m} \geq 0$ and $\sum_{j=0, j \neq i}^{s_{1}} \widehat{\pi}_{i j}=1-\widehat{\pi}_{i i}, \sum_{n=0, n \neq m}^{s_{2}} \widehat{\lambda}_{m n}=1-\widehat{\lambda}_{m m}$ for all $\{i, j\} \epsilon$ $S_{1}$ and $\{m, n\} \in S_{2}$. The transition probability matrices $\hat{\pi} \triangleq$ $\left[\widehat{\pi}_{i j}\right]$ and $\widehat{\pi} \triangleq\left[\widehat{\lambda}_{m n}\right]$ are unknown a priori but belong to the following bounded compact set:

$$
\widehat{\pi}=\pi+\Delta \pi, \quad \hat{\lambda}=\lambda+\Delta \lambda,
$$

where $\pi \triangleq\left[\pi_{i j}\right]\left(i, j \in S_{1}\right)$ and $\lambda \triangleq\left[\lambda_{m n}\right]\left(m, n \in S_{2}\right)$ are known constant matrices. $\Delta \pi \triangleq\left[\Delta \pi_{i j}\right]\left(i, j \in S_{1}\right)$ and $\Delta \lambda \triangleq$ 
$\left[\Delta \lambda_{i j}\right]\left(i, j \in S_{2}\right)$ denote the uncertainty in the transition probability matrices, where $\Delta \pi$ and $\Delta \lambda$ satisfy

$$
\begin{gathered}
\sum_{j=0, j \neq i}^{s_{1}} \Delta \pi_{i j}=-\Delta \pi_{i j} \quad\left(i, j \in S_{1}\right), \\
\sum_{n=0, n \neq m}^{s_{2}} \Delta \lambda_{m n}=-\Delta \lambda_{m n} \quad\left(m, n \in S_{2}\right),
\end{gathered}
$$

where $0 \leq\left|\Delta \pi_{i j}\right| \leq \varepsilon_{i j}, 0 \leq\left|\Delta \lambda_{m n}\right| \leq \varepsilon_{m n}$, and $\varepsilon_{i j}$ and $\varepsilon_{m n}$ are the known small scalar for all $\left(i, j \in S_{1}\right)$ and $\left(m, n \in S_{2}\right)$, respectively.

Remark 1. Closed-loop system (3) is a linear system with the Markovian delays $d(k)$ and $\tau(k)$, which describe the behavior of the S-C and C-A random time delays, and with the uncertain transition probabilities.

Remark 2. The uncertain transition probabilities $\hat{\pi}$ and $\hat{\lambda}$ contain the certain terms $\pi$ and $\lambda$, and the uncertain terms $\Delta \pi$ and $\Delta \lambda$, respectively. The uncertain terms $\Delta \pi$ and $\Delta \lambda$ are bounded, and the sums of the elements in each row are zeros.

\section{Stability Analysis and Controller Design}

By applying a Lyapunov approach and a linear matrix inequality technique, this section provides sufficient conditions for the stochastic stability and the synthesis of state feedback controller design of the system (3).

Definition 3 (see [21]). The closed-loop system (3) is said to be stochastically stable if, for every finite $x_{0}=x(0)$, initial mode $d_{0}=d(0) \in S_{1}$ and $\tau_{0}=\tau(0) \in S_{2}$, there exists a finite $\mathscr{W}>0$ such that

$$
\mathbb{E}\left\{\sum_{k=0}^{\infty}\|x(k)\|^{2} \mid x_{0}, d_{0}, \tau_{0}\right\}<x_{0}^{T} \mathscr{W} x_{0}
$$

Theorem 4. For the system (3), random but bounded scalars $d(k) \in[\underline{d} \bar{d}]$ and $\tau(k) \in[\underline{\tau} \quad \bar{\tau}]$. If, for each mode $\{i, j\} \in S_{1}$ and $\{m, n\} \in S_{2}$ and matrices $P_{i, m}>0, Q_{1}>0, Q_{2}>0, Q_{3}>0$, $R_{1}>0$, and $R_{2}>0, \mathscr{M}_{s}=\left[\begin{array}{llll}\mathscr{M}_{1 s} & \mathscr{M}_{2 s} & \mathscr{M}_{3 s}\end{array}\right]$ and $K$ exist that satisfy the following matrix inequalities:

$$
\Gamma_{i, m}=\left[\begin{array}{ccc}
-R_{1}^{-1} & 0 & \Xi_{1} \\
* & -R_{2}^{-1} & \Xi_{2} \\
* & * & \Xi_{3}
\end{array}\right]<0,
$$

where

$$
\begin{aligned}
& \Xi_{1}=\left[\begin{array}{lllll}
\bar{t}(A-I) & 0 & \bar{t} B K & 0 & 0
\end{array}\right], \\
& \Xi_{2}=[(\bar{t}-\underline{t})(A-I) \quad 0 \quad(\bar{t}-\underline{t}) B K \quad 0 \quad 0], \\
& \Xi_{3}=\mp+\operatorname{sym} \overline{\mathscr{M}}^{T} \Omega \text {, }
\end{aligned}
$$

$$
\Psi=\left[\begin{array}{ccccc}
\Psi_{11} & \bar{P}_{i, m} & R_{1} & 0 & 0 \\
* & \Psi_{22} & 0 & 0 & 0 \\
* & * & \Psi_{33} & R_{1}+R_{2} & R_{2} \\
* & * & * & \Psi_{44} & 0 \\
* & * & * & * & \Psi_{55}
\end{array}\right] \text {, }
$$$$
\Psi_{11}=\bar{P}_{i, m}-P_{i, m}-R_{1}+Q_{1}+Q_{2}+(\bar{t}-\underline{t}+1) Q_{3},
$$$$
\Psi_{22}=\bar{P}_{i, m}, \quad \Psi_{33}=-Q_{3}-2 R_{1}-2 R_{2} \text {, }
$$$$
\Psi_{44}=-Q_{1}-R_{1}-R_{2}, \quad \Psi_{55}=-Q_{2}-R_{2},
$$$$
\overline{\mathscr{M}}=\left[\begin{array}{lllll}
\mathscr{M}_{1 s} & \mathscr{M}_{2 s} & \mathscr{M}_{3 s} & 0 & 0
\end{array}\right],
$$$$
\Omega=\left[\begin{array}{lllll}
A-I & -I & B K & 0 & 0
\end{array}\right],
$$$$
\bar{P}_{i, m}=\sum_{j=0}^{s_{1}} \sum_{n=0}^{s_{2}} \widehat{\pi}_{i j} \widehat{\lambda}_{m n} P_{j, n} \text {, }
$$$$
\bar{t}=\bar{d}+\bar{\tau}, \quad \underline{t}=\underline{d}+\underline{\tau},
$$

and $\hat{\pi}_{i j}$ and $\hat{\lambda}_{m n}$ are defined in (4) and (5).

Then the closed-loop system (3) is stochastically stable.

Proof. For the closed-loop system (3), the stochastic Lyapunov functional candidate is constructed as follows:

$$
V(k)=V_{1}(k)+V_{2}(k)+V_{3}(k)+V_{4}(k),
$$

with

$$
\begin{aligned}
V_{1}(k)= & x(k)^{T} P(d(k), \tau(k)) x(k), \\
V_{2}(k)= & \sum_{l=k-\bar{t}}^{k-1} x(l)^{T} Q_{1} x(l)+\sum_{l=k-\underline{t}}^{k-1} x(l)^{T} Q_{2} x(l), \\
V_{3}(k)= & \sum_{\theta=-\bar{t}+2} \sum_{l=k+\theta-1}^{k-1} x(l)^{T} Q_{3} x(l)+\sum_{l=k-\eta_{k}}^{k-1} x(l)^{T} Q_{3} x(l), \\
V_{4}(k)= & \sum_{\theta=-\bar{t}+1}^{0} \sum_{l=k+\theta-1}^{k-1} \bar{t} \delta(l)^{T} R_{1} \delta(l) \\
& +\sum_{\theta=-\bar{t}+1}^{-t} \sum_{l=k+\theta-1}^{k-1}(\bar{t}-\underline{t}) \delta(l)^{T} R_{2} \delta(l),
\end{aligned}
$$

where $P(d(k), \tau(k))>0, Q_{1}>0, Q_{2}>0, Q_{3}>0, R_{1}>0$, and $R_{2}>0$.

Let $\delta(l)=x(l+1)-x(l)$, noting that $x(k+1)=A x(k)+$ $B K x\left(k-\eta_{k}\right)$. Then $0=(A-I) x(k)-\delta(k)+B K x(k-$ $\left.\eta_{k}\right)$. For simplicity, we will use the following notations: 
$\zeta(k)=\left[\begin{array}{lll}x(k)^{T} & \delta(k)^{T} & x\left(k-\eta_{k}\right)^{T}\end{array}\right]^{T}$. Then, for any weighting matrices $\mathscr{M}_{s}$ with compatible dimensions (and let $\mathscr{M}_{s}=$ $\left.\left[\begin{array}{lll}\mathscr{M}_{1 s} & \mathscr{M}_{2 s} & \mathscr{M}_{3 s}\end{array}\right]\right)$, we have $2 \zeta(k)^{T} \mathscr{M}_{s}^{T}((A-I) x(k)-\delta(k)+$ $\left.B K x\left(k-\eta_{k}\right)\right)=0$. Along the trajectory of the solution of the closed-loop system (3), we obtain

$$
\begin{aligned}
\mathbb{E}\left[\Delta V_{1}(k)\right]= & \mathbb{E}\left\{[x(k)+\delta(k)]^{T} \bar{P}_{i, m}[x(k)+\delta(k)]\right\} \\
& -x(k)^{T} P_{i, m} x(k)+2 \zeta(k)^{T} \mathscr{M}_{s}^{T} \\
& \times\left((A-I) x(k)+B K\left(k-\eta_{k}\right)-\delta(k)\right), \\
\mathbb{E}\left[\Delta V_{2}(k)\right]= & x(k)^{T}\left(Q_{1}+Q_{2}\right) x(k) \\
& -x(k-\bar{t})^{T} Q_{1} x(k-\bar{t}) \\
& -x(k-\underline{t})^{T} Q_{2} x(k-\underline{t}), \\
\mathbb{E}\left[\Delta V_{3}(k)\right] \leq & (\bar{t}-\underline{t}+1) x(k)^{T} Q_{3} x(k) \\
& -x\left(k-\eta_{k}\right)^{T} Q_{3} x\left(k-\eta_{k}\right), \\
\mathbb{E}\left[\Delta V_{4}(k)\right]= & -\bar{t}^{2} \delta(k)^{T} R_{1} \delta(k)+(\bar{t}-\underline{t})^{2} \delta(k)^{T} R_{2} \delta(k) \\
& -\sum_{l=k-\bar{t}}^{k-1} \bar{t} \delta(l)^{T} R_{1} \delta(l) \\
& -\sum_{l=k-\bar{t}}^{k-\underline{t}-1}(\bar{t}-\underline{t}) \delta(l)^{T} R_{2} \delta(l) .
\end{aligned}
$$

By Jensen's inequality, we can obtain

$$
\begin{aligned}
- & \sum_{l=k-\bar{t}}^{k-1} \bar{t} \delta(l)^{T} R_{1} \delta(l) \\
= & -\left(\sum_{l=k-\bar{t}}^{k-\eta_{k}-1}+\sum_{l=k-\eta_{k}}^{k-1}\right)\left(\bar{t}-\eta_{k}+\eta_{k}\right) \delta(l)^{T} R_{1} \delta(l) \\
\leq & -\left(\left(\bar{t}-\eta_{k}\right) \sum_{l=k-\bar{t}}^{k-\eta_{k}-1} \delta^{T}(l) R_{1} \delta(l)+\eta_{k} \sum_{l=k-\eta_{k}}^{k-1} \delta^{T}(l) R_{1} \delta(l)\right) \\
\leq & -\left(\left(\sum_{l=k-\bar{t}}^{k-\eta_{k}-1} \delta(l)\right)^{T} R_{1}\left(\sum_{l=k-\bar{t}}^{k-\eta_{k}-1} \delta(l)\right)\right. \\
& \left.+\left(\sum_{l=k-\eta_{k}}^{k-1} \delta(l)\right)^{T} R_{1}\left(\sum_{l=k-\eta_{k}}^{k-1} \delta(l)\right)\right) \\
= & -\left(x\left(k-\eta_{k}\right)-x(k-\bar{t})\right)^{T} R_{1}\left(x\left(k-\eta_{k}\right)-x(k-\bar{t})\right) \\
& -\left(x(k)-x\left(k-\eta_{k}\right)\right)^{T} R_{1}\left(x(k)-x\left(k-\eta_{k}\right)\right) .
\end{aligned}
$$

Similarly, we have

$$
\begin{aligned}
- & \sum_{l=k-\bar{t}}^{k-t-1}(\bar{t}-\underline{t}) \delta(l)^{T} R_{2} \delta(l) \\
\leq & -\left(\sum_{l=k-\bar{t}}^{k-\eta_{k}-1} \delta(l)\right)^{T} R_{2}\left(\sum_{l=k-\bar{t}}^{k-\eta_{k}-1} \delta(l)\right) \\
& +\left(\sum_{l=k-\eta_{k}}^{k-\underline{t}-1} \delta(l)\right)^{T} R_{2}\left(\sum_{l=k-\eta_{k}}^{k-\underline{t}-1} \delta(l)\right) \\
= & -\left(x\left(k-\eta_{k}\right)-x(k-\bar{t})\right)^{T} R_{2}\left(x\left(k-\eta_{k}\right)-x(k-\bar{t})\right) \\
& -\left(x(k-\underline{t})-x\left(k-\eta_{k}\right)\right)^{T} R_{2}\left(x(k-\underline{t})-x\left(k-\eta_{k}\right)\right) .
\end{aligned}
$$

By substituting (16) and (17) to (15) and then combining (12), (13), and (14), we have

$$
\begin{aligned}
& \mathbb{E}[\Delta V] \\
& \leq \xi(k)^{T}\left\{\Xi_{3}+\left[\begin{array}{lllll}
\bar{t}(A-I) & 0 & \bar{t} B K & 0 & 0
\end{array}\right]^{T}\right. \\
& \times R_{1}\left[\begin{array}{lllll}
\bar{t}(A-I) & 0 & \bar{t} B K & 0 & 0
\end{array}\right]
\end{aligned}
$$

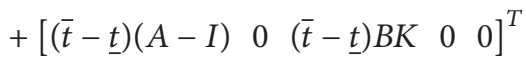

$$
\begin{aligned}
& \left.\times R_{2}[(\bar{t}-\underline{t})(A-I) \quad 0(\bar{t}-\underline{t}) B K \quad 0 \quad 0]\right\} \xi(k) \\
& =\xi(k)^{T} \Gamma_{i, m} \xi(k),
\end{aligned}
$$

where $\xi(k)=\left[\begin{array}{lll}\zeta(k)^{T} & x(k-\bar{t})^{T} x(k-t)^{T}\end{array}\right]^{T}$. By using the Schur complement, (8) guarantees that $\Gamma_{i, m}<0$. Therefore,

$$
\mathbb{E}[\Delta V] \leq-\lambda_{\min }\left(-\Gamma_{i, m}\right) \xi(k)^{T} \xi(k) \leq-\eta x(k)^{T} x(k),
$$

where $\lambda_{\min }\left(-\Gamma_{i, m}\right)$ denotes the minimal eigenvalue of $-\Gamma_{i, m}$ and $\boldsymbol{\eta}=\inf \left\{\lambda_{\min }\left(-\Gamma_{i, m}\right)\right\}$. From (19), it follows that, for any $t>0$,

$$
\mathbb{E}[V(k+1)]-\mathbb{E}[V(0)] \leq-\boldsymbol{\eta} \sum_{k=0}^{t} \mathbb{E}\left[x(k)^{T} x(k)\right] .
$$

Furthermore

$$
\sum_{k=0}^{t} \mathbb{E}\left[x(k)^{T} x(k)\right] \leq \frac{1}{\boldsymbol{\eta}} \mathbb{E}[V(0)] .
$$

By taking $t \rightarrow \infty$ as the limit, we obtain

$$
\sum_{k=0}^{\infty} \mathbb{E}\left[x(k)^{T} x(k)\right] \leq \frac{1}{\boldsymbol{\eta}} \mathbb{E}[V(0)]=\frac{1}{\boldsymbol{\eta}} x_{0}^{T} P\left(d_{0}, \tau_{0}\right) x_{0}<\infty .
$$

According to Definition 3, the closed-loop system (3) exhibits stochastic stability for all uncertain transition probability matrices. 
Theorem 4 gives a sufficient condition for the stochastic stability of the system (3). However, it should be noted that the controller gain $K$ cannot be obtained according to the condition in (8) because of the nonlinear terms $R_{1}^{-1}$ and $R_{2}^{-1}$ and the uncertain terms $\Delta \pi$ and $\Delta \lambda$. To handle this problem, the equivalent LMI conditions are given as follows.

Before proceeding further, we provide the following lemma that will play a significant role in processing the uncertainty terms $\Delta \pi$ and $\Delta \lambda$ of uncertain transition probability matrices $\hat{\pi}$ and $\hat{\lambda}$.

Lemma 5 (see [28]). For any vectors of $a, b \in \mathscr{R}^{n}$ and positive matrix $Z \in \mathscr{R}^{n_{Z}, n_{Z}}$, the following holds:

$$
2 a^{T} b \leq a^{T} Z a+b^{T} Z^{-1} b .
$$

Theorem 6. For the system (3), the random but bounded scalars $d(k) \in[\underline{d} \bar{d}]$ and $\tau(k) \in[\underline{\tau} \bar{\tau}]$. If, for each mode $\{i, j\} \in S_{1}$ and $\{m, n\} \in S_{2}$, the tuning parameters $\varphi_{1}>0$ and $\varphi_{2}>0$, the scalars $\varepsilon_{i j}>0$ and $\varepsilon_{n m}>0$, and matrices $\widehat{P}_{i, m}>0$, $X>0, \widehat{Q}_{1}>0, \widehat{Q}_{2}>0, \widehat{Q}_{3}>0, \widehat{R}_{1}>0, \widehat{R}_{2}>0, Z_{i}>0$, $Z_{m}>0$, and $Z_{j, n}>0$, and $Y$ exist such that

$$
\left[\begin{array}{cccc}
\Theta_{11} & 0 & 0 & \Theta_{14} \\
* & \Theta_{22} & 0 & \Theta_{24} \\
* & * & \Theta_{33} & \Theta_{34} \\
* & * & * & \Theta_{44}
\end{array}\right]<0,
$$

where

$$
\begin{aligned}
& \Theta_{11}=-\operatorname{sym}\{X\}+\widehat{R}_{1}, \quad \Theta_{22}=-\operatorname{sym}\{X\}+\widehat{R}_{2}, \\
& \Theta_{33}=\operatorname{diag}\left\{-\widehat{Z}_{m},-\widehat{Z}_{i},-\widehat{Z}_{j, n}\right\}, \\
& \Theta_{14}=\left[\begin{array}{lllll}
\bar{t}(A X-X) & 0 & \bar{t} B Y & 0 & 0
\end{array}\right],
\end{aligned}
$$

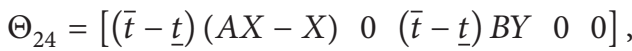

$$
\begin{aligned}
& \Theta_{34}=\left[\begin{array}{lllll}
\Psi_{1} & \Psi_{1} & 0 & 0 & 0 \\
\Psi_{2} & \Psi_{2} & 0 & 0 & 0 \\
\Psi_{3} & \Psi_{3} & 0 & 0 & 0
\end{array}\right], \\
& \widehat{Z}_{m}=\operatorname{diag} \underbrace{\left\{Z_{m}, \ldots, Z_{m}\right\}}_{s_{1}\left(s_{2}-1\right)}, \quad \widehat{Z}_{i}=\operatorname{diag} \underbrace{\left\{Z_{i}, \ldots, Z_{i}\right\}}_{\left(s_{1}-1\right) s_{2}} \text {, } \\
& \widehat{Z}_{j, n}=\operatorname{diag} \underbrace{\left\{Z_{j, n}, \ldots, Z_{j, n}\right\}}_{s_{1} s_{2}}, \\
& \Psi_{1}=\left[\begin{array}{c}
\sqrt{\pi_{i 1}} \Delta \widehat{P}_{1, m} \\
\cdots \\
\sqrt{\pi_{i s_{1}}} \Delta \widehat{P}_{s_{1}, m}
\end{array}\right], \quad \Psi_{2}=\left[\begin{array}{c}
\sqrt{\lambda_{m 1}} \Delta \widehat{P}_{i, 1} \\
\cdots \\
\sqrt{\lambda_{m s_{2}}} \Delta \widehat{P}_{i, s_{2}}
\end{array}\right] \text {, } \\
& \Psi_{3}=\left[\begin{array}{c}
\widehat{P}_{1,1} \\
\cdots \\
\widehat{P}_{s_{1}, s_{2}}
\end{array}\right],
\end{aligned}
$$

$$
\begin{aligned}
& \Delta \widehat{P}_{j, m}=\left[\begin{array}{c}
\widehat{P}_{j, 1}-\widehat{P}_{j, m} \\
\cdots \\
\widehat{P}_{j, m-1}-\widehat{P}_{j, m} \\
\widehat{P}_{j, m+1}-\widehat{P}_{j, m} \\
\cdots \\
\widehat{P}_{j, s_{2}}-\widehat{P}_{j, m}
\end{array}\right], \quad \Delta \widehat{P}_{i, n}=\left[\begin{array}{c}
\widehat{P}_{1, n}-\widehat{P}_{j, n} \\
\cdots \\
\widehat{P}_{i-1, n}-\widehat{P}_{j, n} \\
\widehat{P}_{i+1, n}-\widehat{P}_{j, n} \\
\cdots \\
\widehat{P}_{s_{1}, n}-\widehat{P}_{j, n}
\end{array}\right], \\
& \Theta_{44}=\operatorname{sym}\left\{\Pi^{T} \widehat{\Omega}\right\} \\
& +\left[\begin{array}{ccccc}
\widehat{\Phi}_{11} & \vartheta_{1} \circledast & \vartheta_{2} \widehat{R}_{1} & 0 & 0 \\
* & \vartheta_{1}^{2} \circledast & 0 & 0 & 0 \\
* & * & \vartheta_{2}^{2} \widehat{\mathrm{A}}_{33} & \vartheta_{2}\left(\widehat{R}_{1}+\widehat{R}_{2}\right) & \vartheta_{2} \widehat{R}_{2} \\
* & * & * & \widehat{\Psi}_{44} & 0 \\
* & * & * & * & \widehat{\Phi}_{55}
\end{array}\right], \\
& \widehat{\mathrm{A}}_{11}=\circledast-\widehat{P}_{i, m}-\widehat{R}_{1}+\widehat{Q}_{1}+\widehat{Q}_{2}+(\bar{t}-\underline{t}+1) \widehat{Q}_{3}, \\
& \widehat{\mathrm{A}}_{33}=-\widehat{Q}_{3}-2 \widehat{R}_{1}-2 \widehat{R}_{2}, \quad \widehat{\mathrm{A}}_{44}=-\widehat{Q}_{1}-\widehat{R}_{1}-\widehat{R}_{2} \text {, } \\
& \widehat{4}_{55}=-\widehat{Q}_{2}-\widehat{R}_{2}, \\
& \circledast=\sum_{j=0}^{s_{1}} \sum_{n=0}^{s_{2}}\left(\pi_{i j} \lambda_{m n} \widehat{P}_{j, n}+\frac{\left(\varepsilon_{i j} \varepsilon_{m n}\right)^{2}}{4} Z_{j, n}\right) \\
& +\sum_{j=0}^{s_{1}} \sum_{n=0, n \neq m}^{s_{2}} \frac{\pi_{i j} \varepsilon_{m n}^{2}}{4} Z_{m}+\sum_{j=0, j \neq i}^{s_{1}} \sum_{i=0}^{s_{2}} \frac{\lambda_{m n} \varepsilon_{i j}^{2}}{4} Z_{i}, \\
& \Pi=\left[\begin{array}{lllll}
I & I & I & 0 & 0
\end{array}\right], \\
& \widehat{\Omega}=\left[\begin{array}{lllll}
A X-X & -\vartheta_{1} X & \vartheta_{2} B Y & 0 & 0
\end{array}\right] \text {. }
\end{aligned}
$$

Then the closed-loop system (3) is stochastically stable and the controller $\bar{u}(k)=K \bar{x}(k)=Y X^{-1} \bar{x}(k)$ is a state feedback controller of the system (3).

Proof. Let $\bar{\Delta}_{i}=\operatorname{diag}\left\{I, I, X_{i, m}, \vartheta_{1} X_{i, m}, \vartheta_{2} X_{i, m}, X_{i, m}, X_{i, m}\right\}$, $\mathscr{M}_{1 s}^{-1}=X_{i, m}, \mathscr{M}_{2 s}^{-1}=\vartheta_{1} X_{i, m}$, and $\mathscr{M}_{3 s}^{-1}=\vartheta_{2} X_{i, m}$, where $\vartheta_{1}>0$ and $\vartheta_{2}>0$ are known tuning parameters. We restrict $X_{i, m}$ to be the same for all $\{i, m\}$ (namely, $X_{i, m}=X$ ) and give the notations as

$$
\begin{array}{lll}
\widehat{P}_{i, m}=X^{T} P_{i, m} X, & \widehat{\bar{P}}_{i, m}=X^{T} \bar{P}_{i, m} X, & \widehat{R}_{1}=X^{T} R_{1} X, \\
& \widehat{R}_{2}=X^{T} R_{2} X, & \\
\widehat{Q}_{1}=X^{T} Q_{2} X, & \widehat{Q}_{2}=X^{T} Q_{2} X, & \widehat{Q}_{3}=X^{T} Q_{3} X .
\end{array}
$$

Pre- and postmultiplying $\bar{\Delta}_{i}^{T}$ and $\bar{\Delta}_{i}$ to (8), respectively, we have

$$
\left[\begin{array}{ccc}
-X \widehat{R}_{1}^{-1} X^{T} & 0 & \widehat{\Xi}_{1} \\
* & -X \widehat{R}_{2}^{-1} X^{T} & \widehat{\Xi}_{2} \\
* & * & \widehat{\Xi}_{3}
\end{array}\right]<0,
$$


where

$$
\begin{aligned}
& \widehat{\Xi}_{1}=\left[\begin{array}{lllll}
\bar{t}(A X-X) & 0 & \bar{t} B Y & 0 & 0
\end{array}\right], \\
& \widehat{\Xi}_{2}=[(\bar{t}-\underline{t})(A X-X) \quad 0 \quad(\bar{t}-\underline{t}) B Y \quad 0 \quad 0], \\
& \widehat{\Xi}_{3}=\widetilde{\Phi}+\operatorname{sym}^{T} \widehat{\Omega}, \\
& \widetilde{\Phi}=\left[\begin{array}{ccccc}
\widetilde{\Psi}_{11} & \vartheta_{1} \hat{\bar{P}}_{i, m} & \vartheta_{2} \widehat{R}_{1} & 0 & 0 \\
* & \vartheta_{1}^{2} \hat{\bar{P}}_{i, m} & 0 & 0 & 0 \\
* & * & \vartheta_{2}^{2} \widehat{\Psi}_{33} & \vartheta_{2}\left(\widehat{R}_{1}+\widehat{R}_{2}\right) & \vartheta_{2} \widehat{R}_{2} \\
* & * & * & \widehat{\Psi}_{44} & 0 \\
* & * & * & * & \widehat{\Psi}_{55}
\end{array}\right], \\
& \widetilde{\Phi}_{11}=\widehat{\bar{P}}_{i, m}-\widehat{P}_{i, m}-\widehat{R}_{1}+\widehat{Q}_{1}+\widehat{Q}_{2}+(\bar{t}-\underline{t}+1) \widehat{Q}_{3}, \\
& \bar{P}_{i, m}=\sum_{j=0}^{s_{1}} \sum_{n=0}^{s_{2}} \widehat{\pi}_{i j} \widehat{\lambda}_{m n} \widehat{P}_{j, n}
\end{aligned}
$$

where $\bar{t}$ and $\underline{t}$ are defined in Theorem 4 and $\widehat{4}_{33}, \widehat{\mathrm{f}}_{44}$, and $\widehat{\Phi}_{55}$ are defined in Theorem 6.

According to the assumption on uncertain transition probabilities $\widehat{\pi}$ and $\hat{\lambda}$ and the fact that $\sum_{j=0, j \neq i}^{s_{1}} \Delta \pi_{i j}=-\Delta \pi_{i i}$ and $\sum_{n=0, n \neq m}^{s_{2}} \Delta \lambda_{m n}=-\Delta \lambda_{m m}$, one has

$$
\begin{aligned}
\widehat{\bar{P}}_{i, m}= & \sum_{j=0 n=0}^{s_{1}} \sum^{s_{2}} \widehat{\pi}_{i j} \widehat{\lambda}_{m n} \widehat{P}_{j, n} \\
= & \sum_{j=0 n=0}^{s_{1}} \sum^{s_{2}}\left(\pi_{i j}+\Delta \pi_{i j}\right)\left(\lambda_{m n}+\Delta \lambda_{m n}\right) \widehat{P}_{j, n} \\
= & \sum_{j=0 n=0}^{s_{1}} \sum^{s_{2}} \pi_{i j} \lambda_{m n} \widehat{P}_{j, n}+\sum_{j=0}^{s_{1}} \sum_{n=0}^{s_{2}} \pi_{i j} \Delta \lambda_{m n} \widehat{P}_{j, n} \\
& +\sum_{j=0 n=0}^{s_{1}} \sum_{s_{2}}^{s_{2}} \Delta \pi_{i j} \lambda_{m n} \widehat{P}_{j, n}+\sum_{j=0}^{s_{1}} \sum_{n=0}^{s_{2}} \Delta \pi_{i j} \Delta \lambda_{m n} \widehat{P}_{j, n} .
\end{aligned}
$$

Note that

$$
\begin{aligned}
\sum_{j=0}^{s_{1}} \sum_{n=0}^{s_{2}} \pi_{i j} \Delta \lambda_{m n} \widehat{P}_{j, n} & \\
= & \sum_{j=0}^{s_{1}} \pi_{i j}\left(\sum_{n=0, n \neq m}^{s_{2}} \Delta \lambda_{m n} \widehat{P}_{j, n}+\Delta \lambda_{m m} \widehat{P}_{j, m}\right) \\
& =\sum_{j=0}^{s_{1}} \pi_{i j} \sum_{n=0, n \neq m}^{s_{2}} \Delta \lambda_{m n}\left(\widehat{P}_{j, n}-\widehat{P}_{j, m}\right) .
\end{aligned}
$$

By Lemma 5 and the fact that $\left|\Delta \lambda_{m n}\right| \leq \varepsilon_{m n}$, we have

$$
\begin{aligned}
& \sum_{j=0}^{s_{1}} \sum_{n=0}^{s_{2}} \pi_{i j} \Delta \lambda_{m n} \widehat{P}_{j, n} \\
& \leq \sum_{j=0}^{s_{1}} \pi_{i j} \sum_{n=0, n \neq m}^{s_{2}}\left(\frac{1}{4} \varepsilon_{m n}^{2} Z_{m}+\left(\widehat{P}_{j, n}-\widehat{P}_{j, m}\right)^{T}\right. \\
&\left.\times Z_{m}^{-1}\left(\widehat{P}_{j, n}-\widehat{P}_{j, m}\right)\right) .
\end{aligned}
$$

Similarly, we have

$$
\begin{aligned}
& \sum_{j=0}^{s_{1}} \sum_{n=0}^{s_{2}} \Delta \pi_{i j} \lambda_{m n} \widehat{P}_{j, n} \\
& \leq \sum_{j=0, j \neq i}^{s_{1}} \sum_{n=0}^{s_{2}} \lambda_{m n} \\
& \quad \times\left(\frac{1}{4} \varepsilon_{i j}^{2} Z_{i}+\left(\widehat{P}_{j, n}-\widehat{P}_{i, n}\right)^{T} Z_{i}^{-1}\left(\widehat{P}_{j, n}-\widehat{P}_{i, n}\right)\right) .
\end{aligned}
$$

$$
\begin{aligned}
& \sum_{j=0}^{s_{1}} \sum_{n=0}^{s_{2}} \Delta \pi_{i j} \Delta \lambda_{m n} \widehat{P}_{j, n} \\
& \quad \leq \sum_{j=0}^{s_{1}} \sum_{n=0}^{s_{2}}\left(\frac{1}{4} \varepsilon_{i j}^{2} \varepsilon_{m n}^{2} Z_{j, n}+\widehat{P}_{j, n}^{T} Z_{j, n}^{-1} \widehat{P}_{j, n}\right) .
\end{aligned}
$$

Note that, for any matrix $X$, we have $X W^{-1} X^{T} \geq$ $\operatorname{sym}\{X\}-W$ for $W=\widehat{R}_{1}$ and $W=\widehat{R}_{2}$. Combining (29), (31), and (32) and by the Schur complement, (24) can be yielded easily from (27); this completes the proof of Theorem 6.

\section{Numerical Example}

In this section, we illustrate our results through an example. We apply the results in Theorem 6 to a simple inverted pendulum system [5] shown in Figure 2, which is a two-order unstable system. The state variables are $\left[\begin{array}{ll}\varphi & \dot{\varphi}\end{array}\right]^{T}$, where $\varphi$ is the angular position of the pendulum. The parameters used are $m=0.1 \mathrm{~kg}$ and $L=1 \mathrm{~m}$, without friction surfaces. The sampling time is $T_{s}=0.05 \mathrm{~s}$. The plant matrices are given by

$$
A=\left[\begin{array}{ll}
1.0123 & 0.0502 \\
0.4920 & 1.0123
\end{array}\right], \quad B=\left[\begin{array}{l}
0.0125 \\
0.5020
\end{array}\right] .
$$

We assume that the stochastic Markovian jumping S-C delay $d(k) \in\{0,1\}$ and C-A delay $\tau(k) \in\{0,1,2\}$ and their uncertain transition probability matrices are given as follows:

$$
\begin{array}{ccc}
\pi=\left[\begin{array}{ll}
0.4 & 0.6 \\
0.7 & 0.3
\end{array}\right], & \lambda=\left[\begin{array}{lll}
0.4 & 0.3 & 0.3 \\
0.2 & 0.5 & 0.3 \\
0.4 & 0.2 & 0.4
\end{array}\right], \\
\Delta \pi=\left[\begin{array}{cc}
0.02 & -0.02 \\
-0.01 & 0.01
\end{array}\right], & \Delta \lambda=\left[\begin{array}{ccc}
0.03 & -0.03 & 0 \\
-0.02 & 0.01 & 0.01 \\
-0.03 & 0.02 & 0.01
\end{array}\right] .
\end{array}
$$




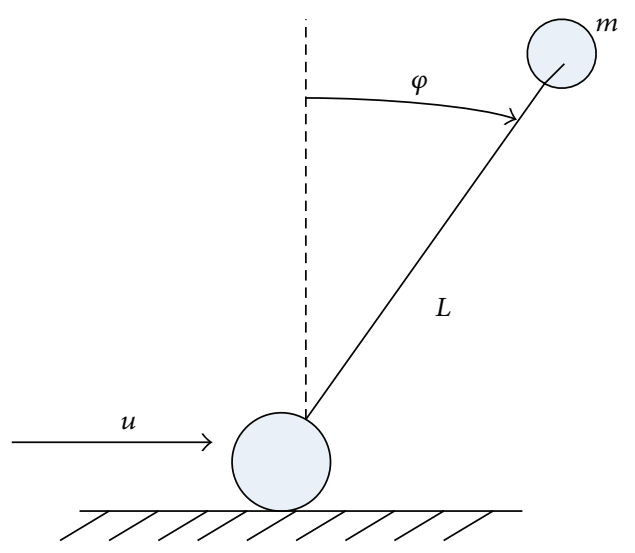

FIGURE 2: A simple inverted pendulum.

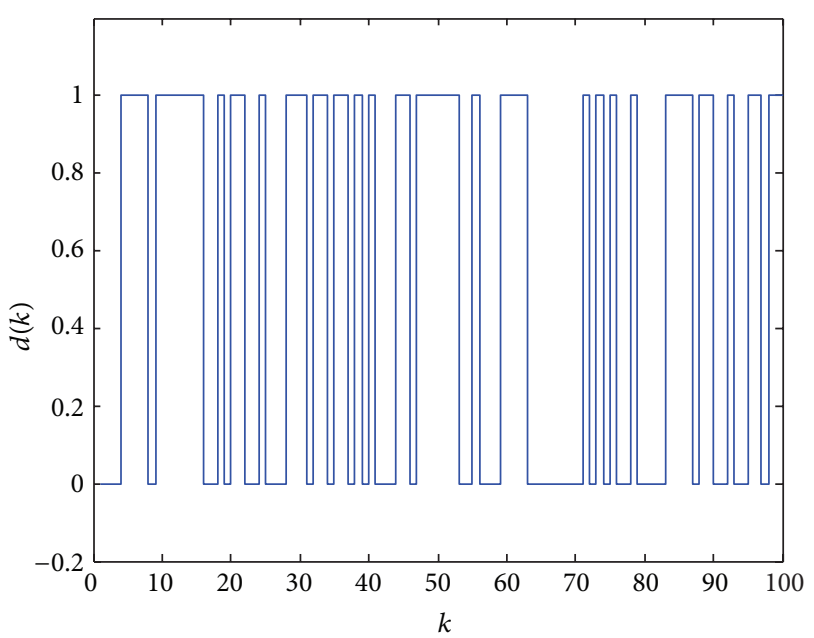

FIGURE 3: Values of the S-C delay $d(k)$.

The eigenvalues of $A$ are 1.1695 and 0.8551 . Therefore, the discrete-time model is unstable.

Figures 3 and 4 show part of the simulation of the Markov chains mode. The initial conditions are as follows: $d(0)=0$, $\tau(0)=0$, and $x(0)=\left[\begin{array}{ll}0.1 & -0.1\end{array}\right]^{T}$. By Theorem 6, when $\varepsilon_{i j}=0.02, \varepsilon_{m n}=0.03, \vartheta_{1}=0.09$, and $\vartheta_{2}=12$, we can obtain the gain matrix $K$ of state feedback controller (2) which is constructed as

$$
\begin{aligned}
K=Y X^{-1} & =\left[\begin{array}{ll}
-0.1046 & -0.1177
\end{array}\right]\left[\begin{array}{cc}
0.1636 & -0.3923 \\
0.3923 & 1.4211
\end{array}\right]^{-1} \\
& =\left[\begin{array}{ll}
-2.4757 & -0.7662
\end{array}\right] .
\end{aligned}
$$

The state trajectories of the system (3) are shown in Figure 5 , where two curves represent state trajectories under the controller gains $K$. Figure 5 also indicates that the system (3) is stochastically stable.

Remark 7. In this example, the uncertain transition probabilities are given as a discrete probability distribution function. When the uncertain transition probability is given as a continuous probability distribution function, we can use the

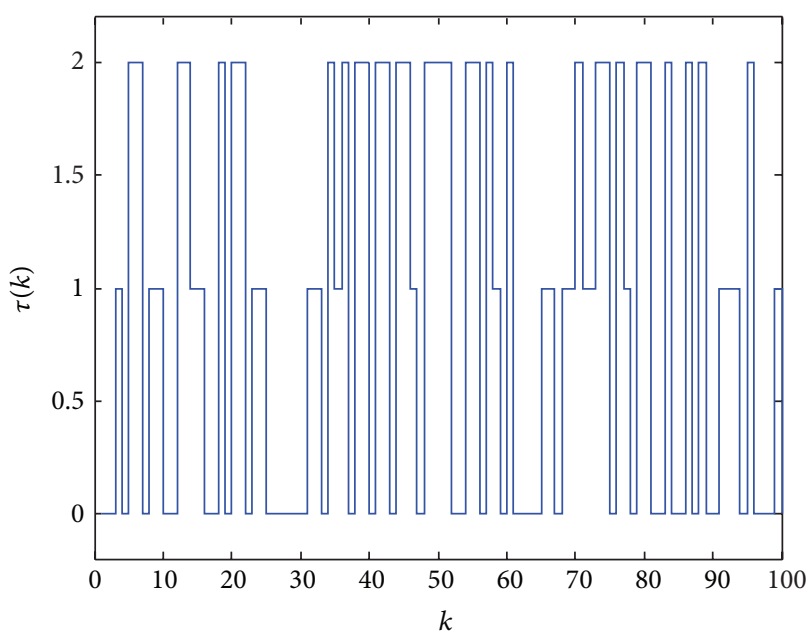

Figure 4: Values of the C-A delay $\tau(k)$.

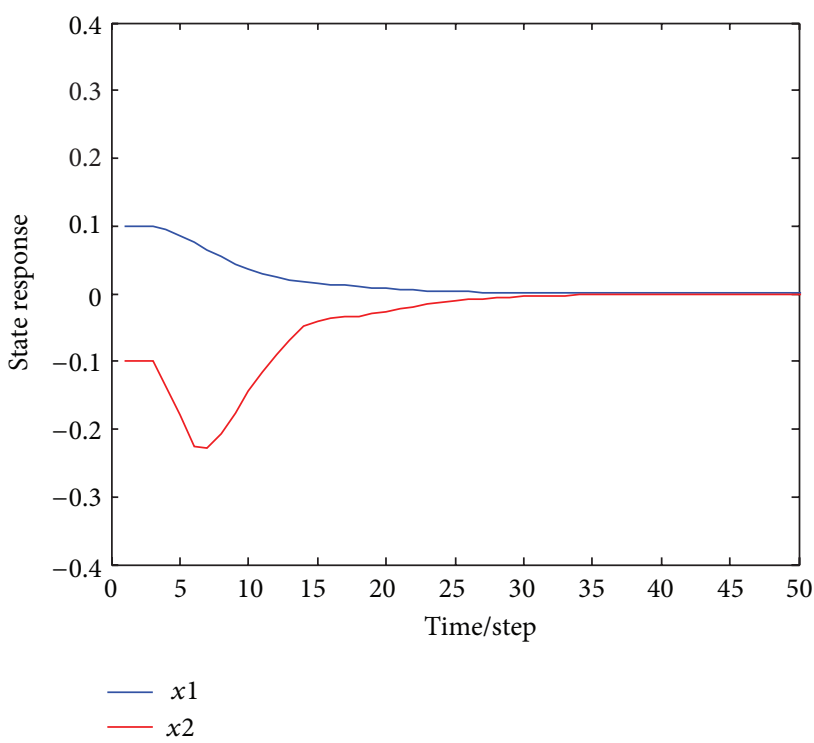

Figure 5: State trajectories under $K$.

$\mathrm{H}_{2}$ norm of the continuous probability distribution function as the upper bound to simulation.

\section{Conclusions}

The state feedback stabilization problem for a class of NCS with the S-C and C-A random time delays is investigated in this paper. The resulting closed-loop NCS is modeled as a linear system with uncertain Markovian transition probabilities. New sufficient conditions on stochastic stability and stabilization are obtained by Lyapunov stability theory and linear matrix inequalities method. An example is presented to illustrate the effectiveness of the approach. Although only the time-delay issue for NCS is addressed in this paper, the method can be extended to the NCS with the random packet dropouts, time delays, and packet dropouts and to the MJLS with the uncertain Markovian transition probabilities. 


\section{Conflict of Interests}

The authors declare that there is no conflict of interests regarding the publication of this paper.

\section{Acknowledgments}

This work was supported partly by the National Natural Science Foundation of China (Grant nos. 61174070 and 61104107), the Natural Science Foundation of Guangdong Province (Grant no. S2013040016183), the Foundation of Key Laboratory of Autonomous Systems and Networked Control, Ministry of Education, China, and the Natural Science Foundation of Shenzhen University (Grant no. 201207).

\section{References}

[1] J. P. Hespanha, P. Naghshtabrizi, and Y. G. Xu, "A survey of recent results in networked control systems," Proceedings of the IEEE, vol. 95, no. 1, pp. 138-162, 2007.

[2] M. C. F. Donkers, W. P. M. H. Heemels, N. van de Wouw, and L. Hetel, "Stability analysis of networked control systems using a switched linear systems approach," IEEE Transactions on Automatic Control, vol. 56, no. 9, pp. 2101-2115, 2011.

[3] H. Li and Y. Shi, "Output feedback predictive control for constrained linear systems with intermittent measurements," Systems and Control Letters, vol. 62, no. 4, pp. 345-354, 2013.

[4] C. Z. Zhang, G. Feng, J. B. Qiu, and Y. Y. Shen, "Control synthesis for a class of linear network-based systems with communication constraints," IEEE Transactions on Automatic Control, vol. 60, no. 8, pp. 3339-3348, 2013.

[5] L. Qiu, B. G. Xu, and S. B. Li, " $H_{2} / H_{\infty}$ control of networked control system with random time delays," Science China: Information Sciences, vol. 54, no. 12, pp. 2615-2630, 2011.

[6] D. Yue, E. G. Tian, Z. D. Wang, and J. Lam, "Stabilization of systems with probabilistic interval input delays and its applications to networked control systems," IEEE Transactions on Systems, Man, and Cybernetics A: Systems and Humans, vol. 39, no. 4, pp. 939-945, 2009.

[7] H. Y. Li, X. J. Jing, and H. R. Karimi, "Output-feedback-based $H_{\infty}$ control for vehicle suspension systems with control delay," IEEE Transactions on Industrial Electronics, vol. 61, no. 1, pp. 436-446, 2014.

[8] R. N. Yang, G. P. Liu, P. Shi, C. Thomas, and M. V. Basin, "Predictive output feedback control for networked control systems," IEEE Transactions on Industrial Electronics, vol. 61, no. 1, pp. 512-520, 2014.

[9] M. B. G. Cloosterman, N. van de Wouw, W. P. M. H. Heemels, and H. Nijmeijer, "Stability of networked control systems with uncertain time-varying delays," IEEE Transactions on Automatic Control, vol. 54, no. 7, pp. 1575-1580, 2009.

[10] X. L. Luan, P. Shi, and F. Liu, "Stabilization of networked control systems with random delays," IEEE Transactions on Industrial Electronics, vol. 58, no. 9, pp. 4323-4330, 2011.

[11] R. Q. Lu, Y. Xu, A. Xue, and J. C. Zheng, "Networked control with state reset and quantized measurements: observer-based case," IEEE Transactions on Industrial Electronics, vol. 60, no. 11, pp. 5206-5213, 2013.

[12] H. Fujioka, "A discrete-time approach to stability analysis of systems with aperiodic sample-and-hold devices," IEEE Transactions on Automatic Control, vol. 54, no. 10, pp. 2440-2445, 2009.
[13] Y. Tang, H. J. Gao, W. Zou, and J. Kurths, "Distributed synchronization in networks of agent systems with nonlinearities and random switchings," IEEE Transactions on Systems, Man, and Cybernetics B: Cybernetices, vol. 43, no. 1, pp. 358-370, 2013.

[14] H. Yu and P. J. Antsaklis, "Event-triggered output feedback control for networked control systems using passivity: achieving $L_{2}$ stability in the presence of communication delays and signal quantization," Automatica, vol. 49, no. 1, pp. 30-38, 2013.

[15] W. P. M. H. Heemels, A. R. Teel, N. van de Wouw, and D. Nešić, "Networked control systems with communication constraints: tradeoffs between transmission intervals, delays and performance," IEEE Transactions on Automatic Control, vol. 55, no. 8, pp. 1781-1796, 2010.

[16] H. L. Dong, Z. D. Wang, and H. J. Gao, “Distributed H filtering for a class of Markovian jump nonlinear time-delay systems over lossy sensor networks," IEEE Transactions on Industrial Electronics, vol. 60, no. 10, pp. 4665-4672, 2013.

[17] X. M. Tang and B. C. Ding, "Model predictive control of linear systems over networks with data quantizations and packet losses," Automatica, vol. 49, no. 5, pp. 1333-1339, 2013.

[18] H. P. Li and Y. Shi, "Network-based predictive control for constrained nonlinear systems with two-channel packet dropouts," IEEE Transactions on Industrial Electronics, vol. 61, no. 3, pp. 1574-1582, 2014.

[19] T. C. Yang, "Networked control system: a brief survey," IEE Proceedings: Control Theory and Applications, vol. 153, no. 4, pp. 403-412, 2006.

[20] L. X. Zhang, H. J. Gao, and O. Kaynak, "Network-induced constraints in networked control systems-a survey," IEEE Transactions on Automatic Control, vol. 9, no. 1, pp. 403-416, 2013.

[21] Y. Shi and B. Yu, "Robust mixed $H_{2} / H_{\infty}$ control of networked control systems with random time delays in both forward and backward communication links," Automatica, vol. 47, no. 4, pp. 754-760, 2011.

[22] O. L. V. Costa and D. Z. Figueiredo, "Stochastic stability of jump discrete-time linear systems with Markov chain in a general borel space," IEEE Transactions on Automatic Control, vol. 59, no. 1, pp. 223-227, 2014.

[23] S. P. He, J. Song, and F. Liu, "Unbiased estimation of Markov jump systems with distributed delays," Signal Processing, vol. 100, pp. 85-92, 2014.

[24] S. P. He and F. Liu, "Finite-time $H_{\infty}$ control of nonlinear jump systems with time-delays via dynamic observer-based state feedback," IEEE Transactions on Fuzzy Systems, vol. 20, no. 4, pp. 605-614, 2012.

[25] S. P. He, "Resilient $L_{2}-L_{\infty}$ filtering of uncertain Markovian jumping systems within the finite-time interval," Abstract and Applied Analysis, vol. 2013, Article ID 791296, 7 pages, 2013.

[26] S. P. He and F. Liu, "Adaptive observer-based fault estimation for stochastic Markovian jumping systems," Abstract and Applied Analysis, vol. 2012, Article ID 176419, 11 pages, 2012.

[27] S. P. He and F. Liu, "Robust finite-time estimation of Markovian jumping systems with bounded transition probabilities," Applied Mathematics and Computation, vol. 222, pp. 297-306, 2013.

[28] Y. S. Moon, P. Park, W. H. Kwon, and Y. S. Lee, "Delay-dependent robust stabilization of uncertain state-delayed systems," International Journal of Control, vol. 74, no. 14, pp. 1447-1455, 2001. 


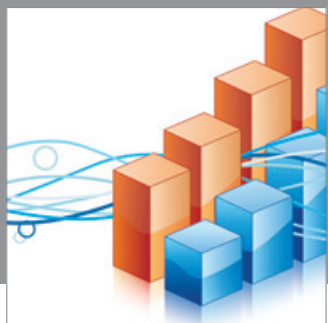

Advances in

Operations Research

mansans

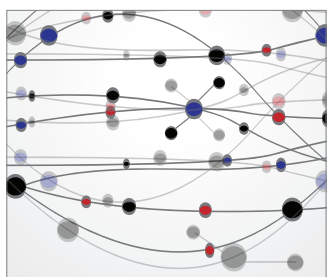

The Scientific World Journal
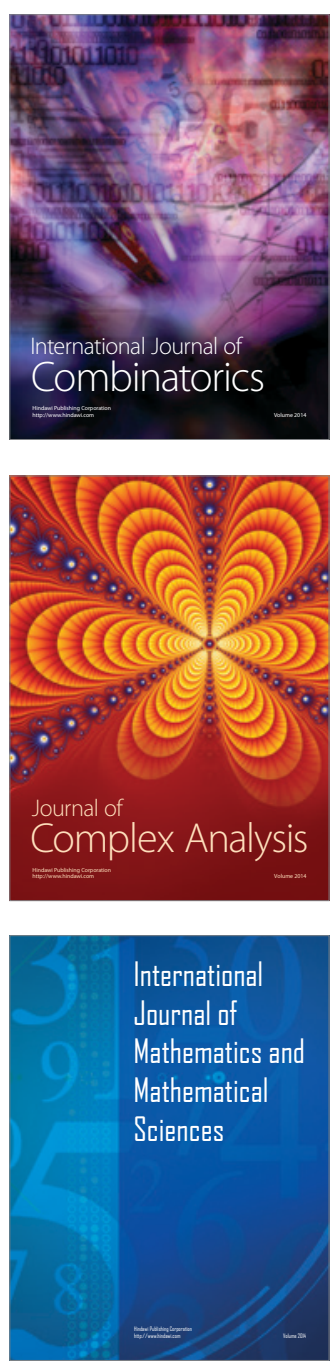
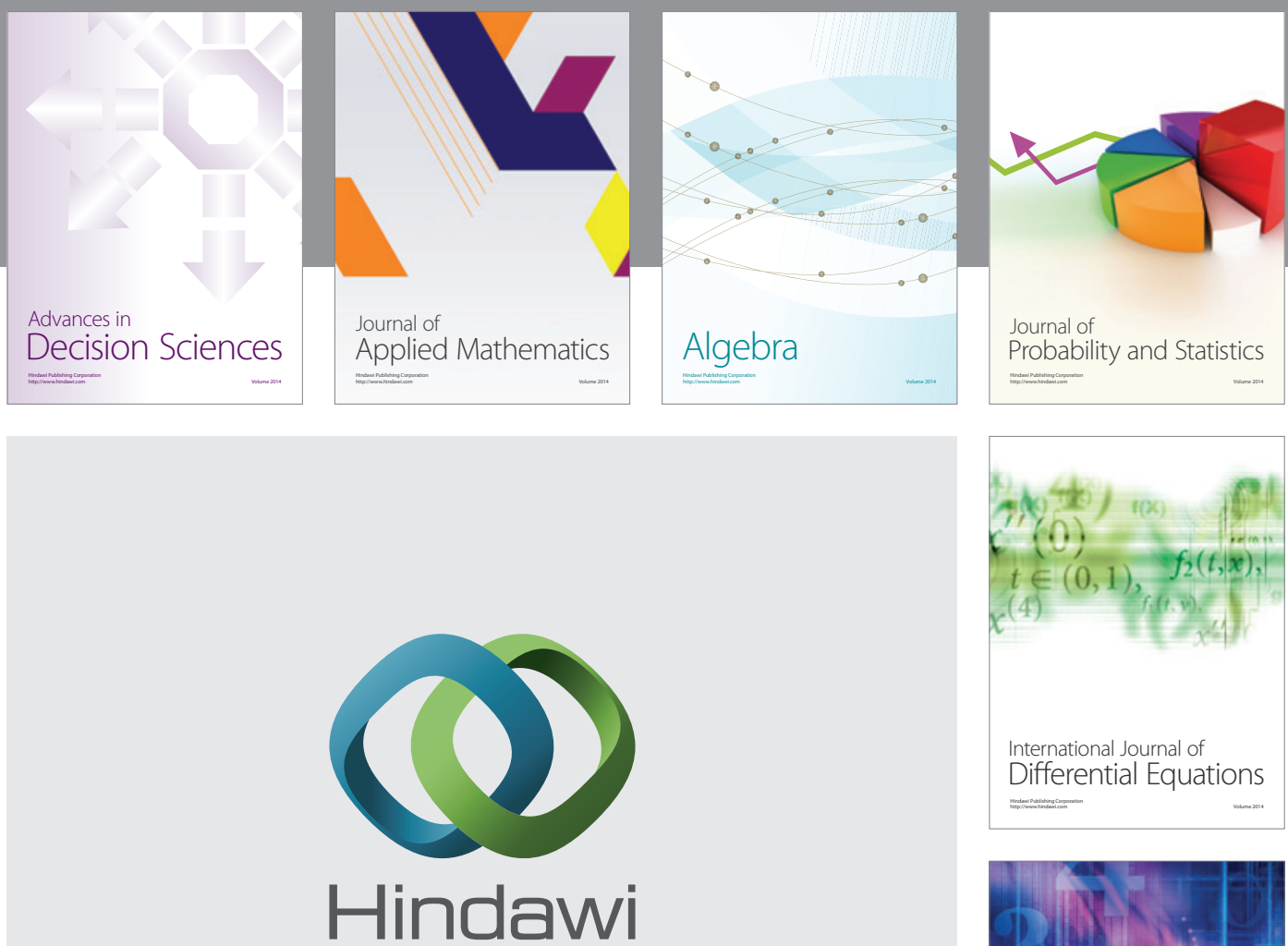

Submit your manuscripts at http://www.hindawi.com
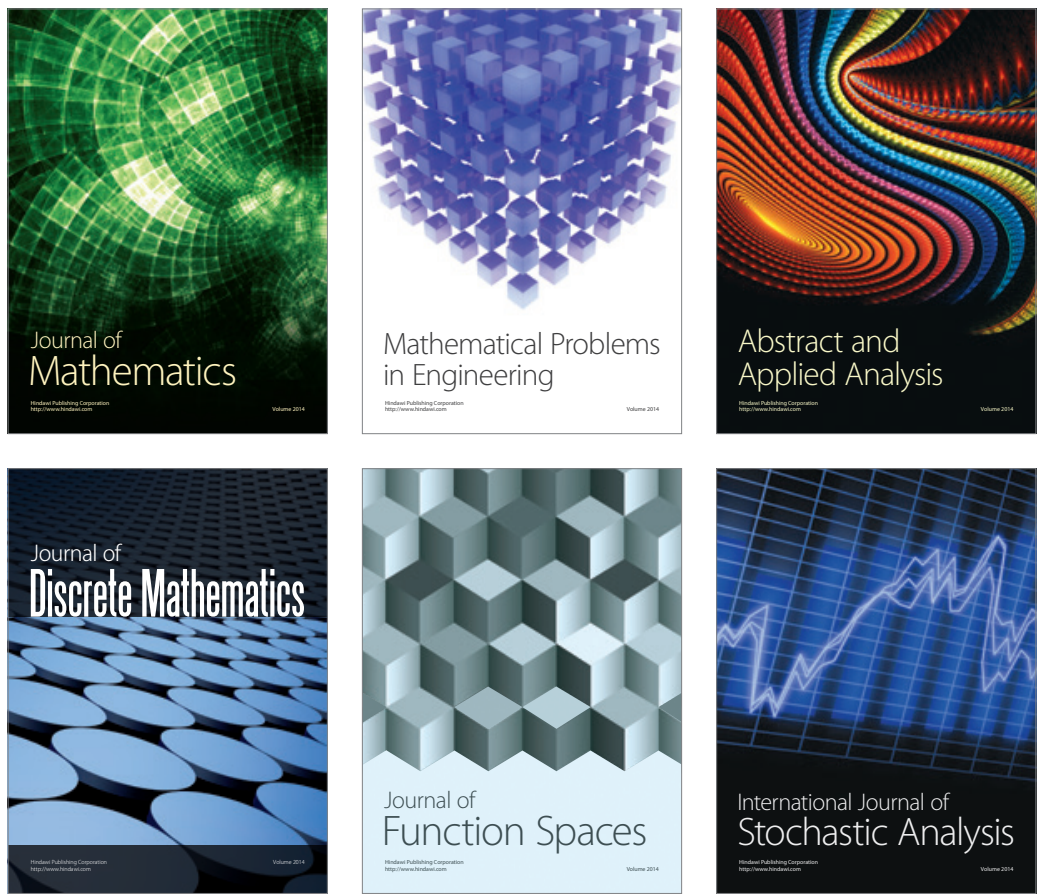

Journal of

Function Spaces

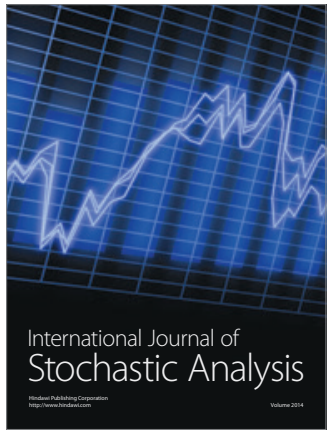

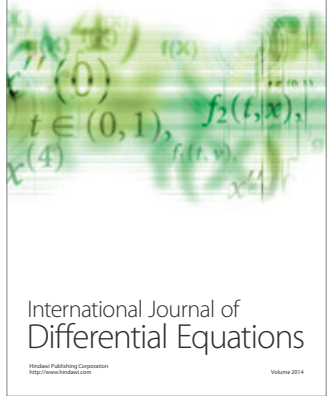
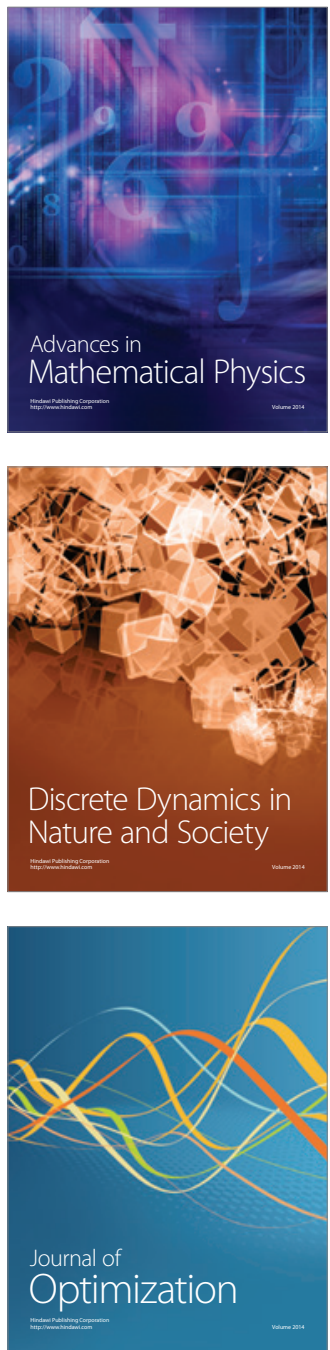